\title{
Description de Rhipicephalus camicasi n. sp. (Acariens, Ixodida) des steppes subdésertiques de la plaine afar
}

\author{
par P. C. MOREL $(*)$, J. MOUCHET $(* *)$ et F. RODHAIN $(* * *)$
}

\section{RÉSUMÉ}

\begin{abstract}
Rhipicephalus camicasi $n$. sp., parasite du bétail et des lièvres dans les steppes xérophytes somaliennes, appartient au groupe de $R h$. sanguineus; seuls les adultes en sont connus.
\end{abstract}

Un certain nombre d'exemplaires d'un Rhipicephalus proche de $R h$. sanguineus a été récolté à 2 reprises, en mai 1969 (J. MOUCHET) et en décembre 1974 (F. RHODHAIN). Il a été confirmé à la suite de la deuxième série de récoltes qu'il s'agit d'une espèce nouvelle, caractérisée principalement par la morphologie du gonopore femelle, et endémique des steppes subdésertiques somaliennes dans la plaine afar.

\section{DESCRIPTION}

Le type mâle et l'allotype femelle proviennent de Randa, sur mouton (04/05/69).

Mâle (fig. 1, A-D)

Caractéristiques générales des espèces du groupe de $R h$. sanguineus.

Basis capituli plus de 2 fois plus large que longue; angle de l'auricule droit, au niveau du tiers antérieur de la longueur de la basis; cornes basidorsales moyennement saillantes, quelques soies sur le bord antérieur de l'auricule.

Conscutum à fosses peu profondes, de tégument ridé, la médiane en ovale allongé, les paramédianes larges et ramassées; sillon scapulaire inapparent, marqué par un alignement

(*) I. E. M. V. T., 94700 Maisons-Alfort.

(**) O. R. S. T. O. M., 93140 Bondy.

(***) Institut Pasteur, Ecologie virale, 75015 Paris. de ponctuations pilifères grandes; sillon marginal bien dessiné, longé intérieurement de ponctuations pilifères moyennes ou grosses, non contiguës, et délimitant les 2 premiers festons; séries paramédianes et paramarginales de ponctuations pilifères de taille moyenne, bien marquées; ponctuations porales interstitielles petites ou très petites, laissant paraître le tégument du conscutum brillant et presque lisse entre les séries paramédianes et paramarginales de ponctuations pilifères.

Feston médian pouvant faire saillie sous forme de mamelon, muni d'un sclérite basal dorsal, mais sans sclérite terminal en croissant ; épines des coxae du type de celles de $R h$. sanguineus; coxa 1 sans saillie antérieure dépassant l'angle scapulaire du conscutum en vue dorsale; articles fémoraux, génuaux et tibiaux des pattes III et IV modérément élargis; plaques anales en battoirs, à angle interne marqué, antérieur au niveau de l'angle externe arrondi ; stigmates à portion caudale de faible largeur, mais non effilée.

\section{Femelle (fig. 1, E-I)}

Basis capituli plus de 2 fois plus large que longue: angle de l'auricule droit, au niveau du tiers antérieur de la basis; quelques soies (2-4) sur le bord antérieur de l'auricule; aires poreuses petites, ovalaires, distantes.

Scutum plus long que large, à sillon scapulaire marqué, bordé intérieurement de ponctuations 

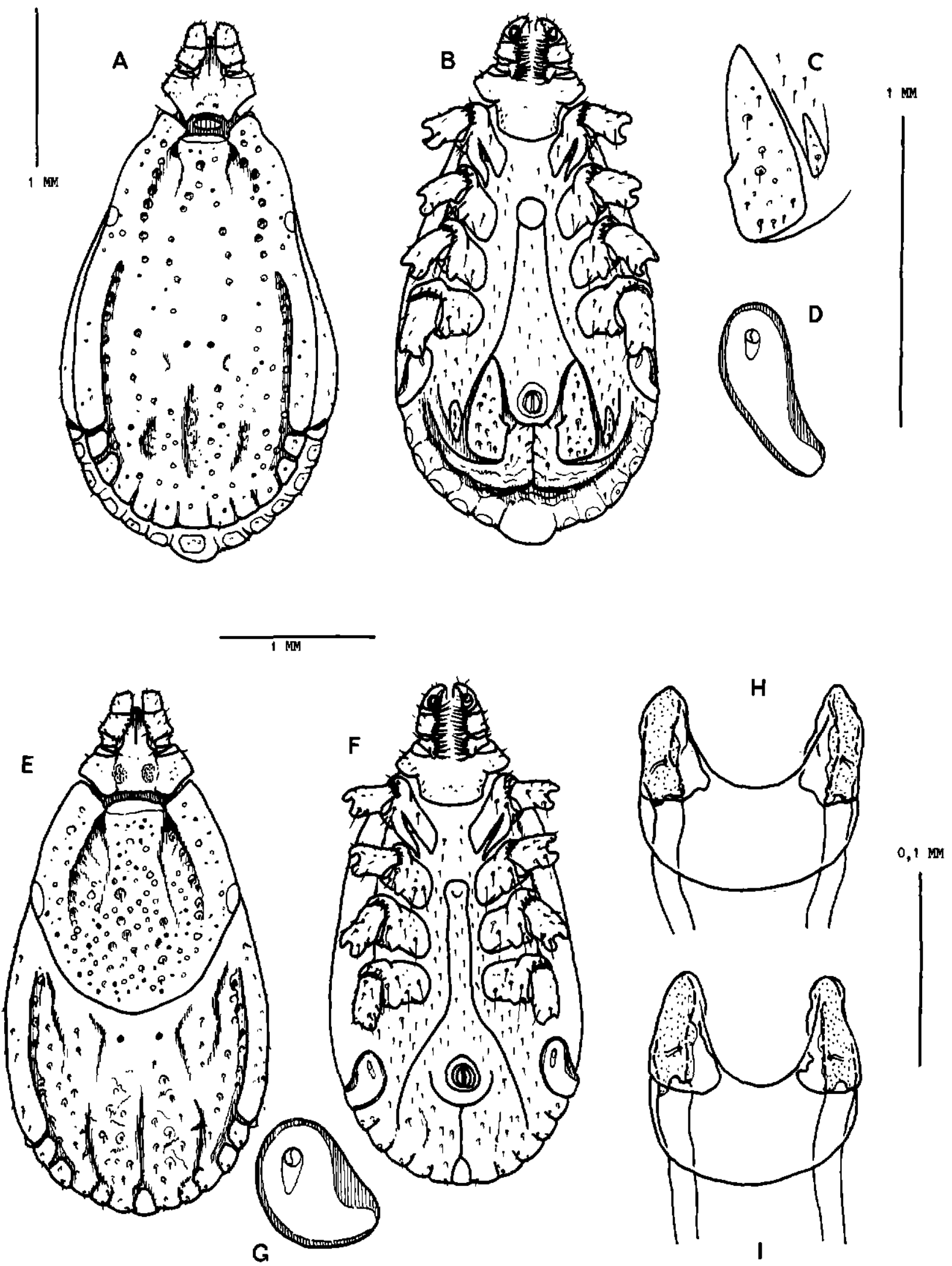

Fig. 1 - Rhipicephalus aamicasi

(exemplaires de Randa, Afars \& Issas) 
pilifères moyennes ; ponctuations porales interstitielles petites, laissant ressortir les ponctuations pilifères moyennes ou grosses du champ cervical. Alloscutum pourvu de soies courtes, cylindriques ou effilées.

Coxae et épines coxales du type de celles de $R h$. sanguineus. Sclérites de l'atrium gonoporal dans le prolongement des parois latérales du vagin, massifs et très faiblement convexes, légèrement pigmentés; il n'y a pas d'élargissement du conduit génital femelle au niveau de l'atrium; les sclérites de l'atrium présentent des expansions ventrales non pigmentées atteignant le niveau de la lèvre du gonopore.

\section{COMMENTAIRES}

Le mâle de $R h$. camicasi se distingue de $R h$. sanguineus, comme toutes les espèces africaines du groupe, par l'élargissement caudal non effilé du stigmate; cet élargissement est pourtant moins important que chez $R h$. guilhoni, $R h$. sulcatus ou $R h$. bergeoni; l'absence de tégument chagriné sur le conscutum distingue $R h$. bergeoni de tous les mâles des autres espèces du groupe.

Les sclérites du gonopore femelle de $R h$. sulcatus et $R h$. bergeoni sont très massifs et présentent une expansion ventrale large et très pigmentée. La transparence de cette expansion chez $R$ h. camicasi fait qu'elle est souvent difficile à observer en début d'éclaircissement, et que la morphologie de l'atrium évoque alors plutôt $R h$. sanguineus ou $R h$. guilhoni; or, chez ces derniers, l'atrium gonoporal est très élargi par rapport à la largeur du vagin, et présente donc un aspect très globuleux en cupule, avec des sclérites nettement convexes latéralement (cf. fig. 2) ; chez la femelle de Rh. guilhoni, par ailleurs, la lèvre du gonopore présente des marges hyalines assez larges.

La nouvelle espèce est dénommée en hommage amical à J. L. CAMICAS, renommé pour ses études sur les Haemaphysalis africaines du groupe de Hm. leachi.
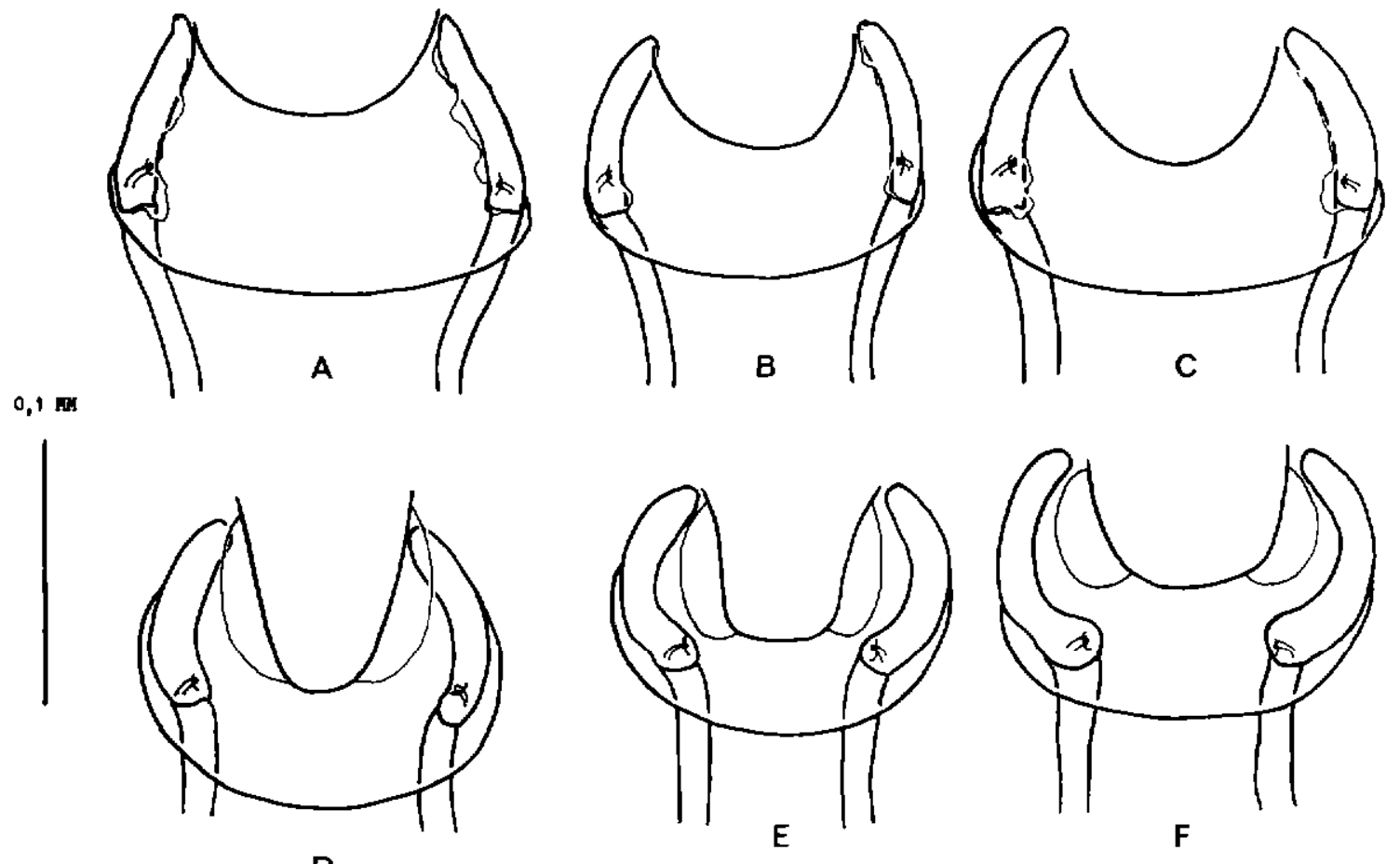

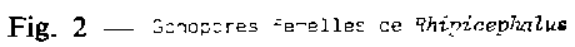

$$
\begin{aligned}
& \text { Rh.sanguzneus } A \text { : Atar ("iasitanis) : Zaraechinus aethiopious (11/48) } \\
& \text { B : Agajes (iger) : Vhipes paltida (29/07/47) } \\
& \text { C : Lekar (Sénégal) · ch1en (15/11/61) } \\
& \text { Shiguithonti D : Nioro (Mals): moutan (14/05/55) } \\
& \text { E : Carfur [Soudan) : mouton (30/05/53, H.Hoogstraal) } \\
& \text { F : Kafr el Sneikh (Egypte) (03/07/56, H.Hoogstraal) }
\end{aligned}
$$




\section{DISTRIBUTION}

\section{Ethiopie}

Références originales (1976). HARRAR, Hai Dora: zébu (1 F, 28/07/66). SHOA parc Awash : Lepus capensis (2 MM, 1 F, 25/01/76, J. Thal).

\section{Afars et Issas}

Références originales. Bankwale: zébu (9 MM, 4 FF, 04/12/74); Assa Geila : mouton (1 F, 15/12/74); Medeho : chèvre (1 F, 09/12/74); Randa: mouton (4 MM, $5 \mathrm{FF}, 04 / 05 / 69$ ); Obok : Lepus capensis (1 F, 12/69).

HOOGSTRAAL (1953, Rh. sanguineus p. p.). Ali Sabiet $(07 / 50)$ : dromadaire (1 M), mouton $(1 \mathrm{M})$, chèvre $(1 \mathrm{M})$; Dikhil $(07 / 50:$ mouton ( $5 \mathrm{MM}, 4 \mathrm{FF}$ ), chèvre (4 MM, $7 \mathrm{FF}$ ), Lepus capensis $(1 \mathrm{M})$.

Rh. camicasi doit vraisemblablement exister au Somali sur la côte du golfe d'Aden; il est possible qu'il soit également établi sur la côte de la Mer Rouge, notamment en Erythrée; peut-être plus au nord, au Soudan et en Egypte.

\section{BIOLOGIE}

Du fait de ses affinités avec les Rhipicephalus du groupe $R h$. sanguineus, il y a tout lieu de supposer que le cycle de $R h$. camicasi est trixène ditrope, faisant intervenir pour les repas des larves et des nymphes les rongeurs myomorphes des steppes.

\section{HABITAT}

Les rares récoltes de $R h$. camicasi sont en rapport avec les steppes subdésertiques somaliennes de la plaine afar. C'est donc un élément caractéristique de cette zone du point de vue de la faune, avec Hyalomma erythraeum.

\section{SUMMARY}

Description of Rhipicephalus camicasi n. sp. (Acaridae, Ixodida) of Afar plain subdesert steppes

Rhipicephalus camicasi n. sp., a parasite of cattle and hares in the subdesert somalian steppe, belongs to the $R h$. sanguineus group, its adults only are known.

\section{RESUMEN}

Descripción de Rhipicephalus camicasi n. sp. (Acaridos, Ixodida) de las estepas subdesérticas de la llanura Afar

Rhipicephalus camicasi n. sp. paràsito del ganado y de las liebres en las estepas xerofitas somalienses, pertenece al grupo de $R h$. sanguineus; solo se conocen los adultos.

\section{BIBLIOGRAPHIE}

HOOGSTRAAL (H.). On ticks (lxodidae) of Southern French Somaliland and the rediscovery of Rhipicephalus longicoxatus Neumann, 1905. Ann. ent. Soc. Am., 1953, 46 (3) : 393-398.
MOREL (P. C.) et VASSILIADES (G.). Les Rhipicephalus du groupe sanguneus : espèces africaines (Acariens, Ixodidae). Rev. Elev. Méd. vét. Pays trop., 1962, 15 (4) : 343-386. 